\title{
Scaling of spin relaxation and angular momentum dissipation in permalloy nanowires
}

\author{
T. A. Moore, ${ }^{1, *}$ M. Kläui,,${ }^{1}$ L. Heyne, ${ }^{1}$ P. Möhrke, ${ }^{1}$ D. Backes,,${ }^{1 \dagger}$ J. Rhensius,,${ }^{1,}$ U. Rüdiger, ${ }^{1}$ L. J. Heyderman, ${ }^{2}$ J.-U. Thiele, ${ }^{3}$ \\ G. Woltersdorf, ${ }^{4}$ C. H. Back, ${ }^{4}$ A. Fraile Rodríguez, ${ }^{5}$ F. Nolting, ${ }^{5}$ T. O. Mentes, ${ }^{6}$ M. Á. Niño, ${ }^{6}$ A. Locatelli, ${ }^{6}$ A. Potenza, ${ }^{7}$ \\ H. Marchetto, ${ }^{7}$ S. Cavill, ${ }^{7}$ and S. S. Dhesi ${ }^{7}$ \\ ${ }^{1}$ Fachbereich Physik, Universität Konstanz, Universitätsstrasse 10, 78457 Konstanz, Germany \\ ${ }^{2}$ Laboratory for Micro- and Nanotechnology, Paul Scherrer Institut, 5232 Villigen PSI, Switzerland \\ ${ }^{3}$ Hitachi Global Storage Technologies, San Jose Research Center, San Jose, California 95135, USA \\ ${ }^{4}$ Fakultät für Physik, Universität Regensburg, Universitätsstrasse 31, 93040 Regensburg, Germany \\ ${ }^{5}$ Swiss Light Source, Paul Scherrer Institut, 5232 Villigen PSI, Switzerland \\ ${ }^{6}$ Sincrotrone Trieste, Basovizza, 34012 Trieste, Italy \\ ${ }^{7}$ Diamond Light Source Ltd., Harwell Science and Innovation Campus, Didcot, Oxfordshire OX11 ODE, United Kingdom
}

(Received 23 September 2009; published 14 October 2009)

\begin{abstract}
We study the relationship between the damping $(\alpha)$ and the nonadiabaticity of the spin transport $(\beta)$ in permalloy nanowires. $\alpha$ is engineered by Ho doping, and from the characteristics of the current-induced domain-wall velocity, determined by high-resolution x-ray magnetic circular-dichroism photoemission electron microscopy, $\beta$ due to spin relaxation is measured. We find that $\beta$ scales with $\alpha$ and conclude that the spin relaxation that leads to nonadiabatic spin torque originates from the same underlying mechanism as the angular momentum dissipation that causes viscous damping.
\end{abstract}

DOI: 10.1103/PhysRevB.80.132403

PACS number(s): 75.75.+a, 72.25.Ba, 75.60.Ch

Magnetic domain-wall (DW) propagation by spinpolarized current ${ }^{1}$ has attracted increasing attention in the last few years due to fundamental interest in the interaction between current and magnetization, ${ }^{2,3}$ and the possibility of applications in spintronics. ${ }^{4}$ However, despite a number of experimental $^{5-11}$ and theoretical investigations, ${ }^{12-14}$ the mechanism of current-induced DW motion in magnetic nanowires is not fully understood. In a phenomenological approach, two spin-torque terms were added to the LandauLifshitz and Gilbert equation of magnetization dynamics to describe the influence of a current: ${ }^{12,13}$

$$
\dot{\mathbf{m}}=\gamma \mathbf{H} \times \mathbf{m}+\alpha \mathbf{m} \times \dot{\mathbf{m}}-(\mathbf{u} \cdot \nabla) \mathbf{m}+\beta \mathbf{m} \times[(\mathbf{u} \cdot \nabla) \mathbf{m}] .
$$

Here the first and second terms describe the precession and damping of a magnetic moment $\mathbf{m}$ in a magnetic field $\mathbf{H}$ with $\gamma$ as the gyromagnetic ratio and $\alpha$ as the Gilbert damping constant. The third and fourth terms, denoted the adiabatic and nonadiabatic spin torque, respectively, ${ }^{12}$ account for the two possible directions of the spin torque acting on $\mathbf{m}$ with $\mathbf{u}$ an effective DW velocity equal to $\left(P g \mu_{B} / 2 e M_{S}\right) \mathbf{j}$, where $P$ is the spin polarization, $g$ is the Landé factor, $\mu_{B}$ is the Bohr magneton, $e$ is the electron charge, $M_{S}$ is the saturation magnetization, and $\mathbf{j}$ is the current density. The adiabatic spin torque arises when the conduction electron spins follow the spatially varying magnetization within the DW as they travel through it. Conservation of angular momentum then dictates that the electron spins exert a torque on the magnetization, leading to DW motion. The nonadiabatic spin torque (" $\beta$ term") was studied theoretically in detail by Tatara et al., ${ }^{14}$ who split it into two components: (i) a deviation of the electron spin from perfect adiabaticity as a result of spin relaxation and (ii) a nonadiabaticity arising from the rapidly varying magnetization direction (which can be neglected for the wide domain walls investigated here ${ }^{15}$ ). Following, ${ }^{14}$ we as- sociate the parameter $\beta$ only with the nonadiabaticity due to spin relaxation.

The $\beta$ term and its relation to the damping $\alpha$ is key to understanding current-induced DW motion. The ratio $\beta / \alpha$ is predicted to control the nature of the DW motion ${ }^{12,13}$ and is the subject of much debate..$^{3,16-18}$ The discussion about $\beta / \alpha$ is connected to the question of whether Landau-Lifshitz or Gilbert damping provides the more natural description of dissipative magnetization dynamics. ${ }^{3,16}$ This is because, for the special case $\alpha=\beta$, Eq. (1) can be rewritten in a form with Landau-Lifshitz damping. However, it seems unlikely that $\alpha$ is always identical to $\beta$ since their ratio is expected to depend sensitively on material and sample properties, e.g., details of the band structure. ${ }^{3,19}$ It is also predicted that $\alpha$ and $\beta$ scale similarly with the strength of spin-dephasing processes. $^{3,19}$ From experiments it has been deduced that $\beta \neq \alpha$ for wide vortex-type DWs in permalloy wires ${ }^{10}$ as well as for narrow Bloch-type DWs in Co/Pt multilayers. ${ }^{20}$

In this paper we investigate the link between $\alpha$ and $\beta$ by varying the damping and observing the effect on the nonadiabaticity. We engineer $\alpha$ by doping permalloy $\left(\mathrm{Py}, \mathrm{Ni}_{80} \mathrm{Fe}_{20}\right)$ nanowires with Ho (Ref. 21) and then measure the domain-wall velocity $v$ induced by current. Since $v \propto(\beta / \alpha) j$ below Walker breakdown, ${ }^{12,13}$ we obtain insight into $\beta / \alpha$. To understand the effect of varying $\alpha$ when spin torques are absent, we also study field-induced DW motion under zero current.

Py zigzag wires of 1500-nm-width, 20-nm thickness, and length approximately $80 \mu \mathrm{m}$ were fabricated by $e$-beam lithography and lift off on Si. The Py was codeposited with Ho to give five sets of nanowires of different composition: pure Py, and Py doped with 1, 2, 4, and 10 at. $\%$ Ho. $\alpha$ in the nanowires was measured by ferromagnetic resonance to be $0.008,0.02,0.033,0.087$, and 0.26 , respectively. A reduction in $M_{S}(5 \%$ per at. $\% \mathrm{Ho})$ was also measured. ${ }^{22}$

DWs were positioned at the kinks in the nanowire by applying a magnetic field in plane and perpendicular to the 


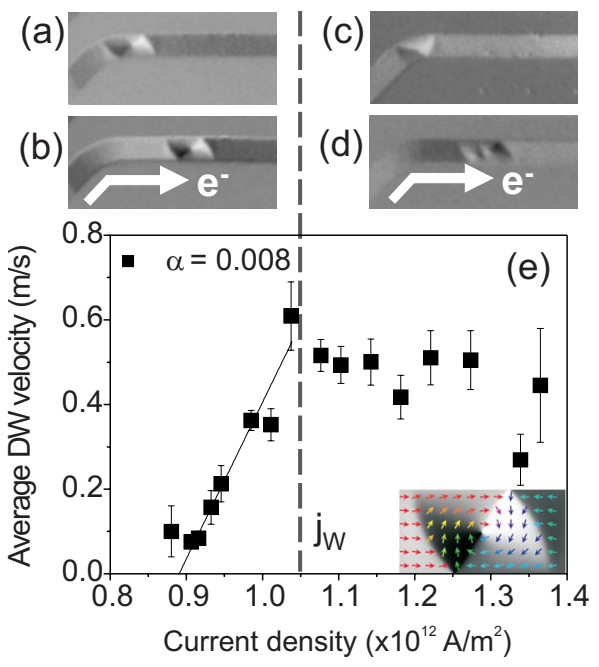

FIG. 1. (Color online) XMCD-PEEM images taken [(a) and (c)] before and [(b) and (d)] after a current pulse of duration $25 \mu$ s and density $j \sim 10^{12} \mathrm{~A} / \mathrm{m}^{2}$. For $j<1.05 \times 10^{12} \mathrm{~A} / \mathrm{m}^{2}$, the DW motion occurs without spin-structure transformations. For $j>1.05$ $\times 10^{12} \mathrm{~A} / \mathrm{m}^{2}$, vortex-core nucleation and annihilation, and propagation of multivortex walls occurs. (e) Average DW velocity $v$ as a function of current density $j$ determined by XMCD-PEEM imaging, for 1500-nm-wide, 20-nm-thick Py wires. Below the Walker threshold current density $j_{W}$ the data are fitted by Eq. (2). The inset is a simulation of a vortex wall in a Py wire with contrast equivalent to the XMCD-PEEM images (a) and (b).

wire direction, as in (Refs. 8 and 10). The lowest energy spin structure for this wire geometry is the vortex wall (VW) with a width on the order of a few hundred $\mathrm{nm}$ and while transverse DWs (TW) can arise directly after initialization they relax immediately to the vortex type as soon as currents are injected or fields applied. ${ }^{11}$ For the measurement of currentinduced DW motion, we used direct imaging by x-ray magnetic circular-dichroism photoemission electron microscopy (XMCD-PEEM), which allows us to determine the wall spin structure and enables comparison with earlier measurements. ${ }^{8,10}$ Images were recorded before and after a current pulse, as in Figs. 1(a)-1(d) and the average $v$ calculated by dividing the distance traveled by the duration of the pulse. For studying field-induced DW motion, the XMCDPEEM is not appropriate since we cannot apply fields inside this setup. Instead we resorted to a time-resolved magnetooptic Kerr-effect (MOKE) technique. The DW velocity is obtained by analyzing the Kerr signal from a focused laser spot on a Py wire, as in (Ref. 23).

After positioning DWs, $25 \mu$ s current pulses were injected with a current density $j$ up to $1.4 \times 10^{12} \mathrm{~A} / \mathrm{m}^{2}$. The spin structure was imaged at room temperature after each pulse. We observe that the current pulses displace the DWs in the direction of the electron flow, and that, depending on $j$, the DW spin structure transforms. Between $\sim 150$ and 500 individual DW movements were analyzed for each level of Ho doping, for a range of $j$. Figure 1(e) shows the average $v(j)$ for pure Py. The threshold current density $j_{c}$ for DW motion is $\sim 0.9 \times 10^{12} \mathrm{~A} / \mathrm{m}^{2}$ and subsequently $v$ increases linearly with $j$ up to a critical value at $\sim 1.05 \times 10^{12} \mathrm{~A} / \mathrm{m}^{2}$. In this region the initial DW spin structures are either TW (which relax immediately to VW on current injection) or VW and the DW motion occurs without modification of the VW structure. For $j>1.05 \times 10^{12} \mathrm{~A} / \mathrm{m}^{2}$ the $\mathrm{DW}$ velocity remains approximately constant within the experimental error up to the maximum $j$ and in this region the DW motion involves spin structure changes, either transformation of a VW to another VW with opposite circulation, transformation of a VW to a multivortex wall or vice versa, or translation of a multivortex wall. These DW spin-structure changes are consistent with previous observations of current-induced VW motion in $1 \mu \mathrm{m}$-wide, 28 -nm-thick Py wires, ${ }^{8}$ where vortices were found to periodically nucleate and annihilate due to spin torque, and sample heating was ruled out as a likely cause of the DW transformations. Since the critical value of $j$ at $\sim 1.05 \times 10^{12} \mathrm{~A} / \mathrm{m}^{2}$ corresponds to the onset of $\mathrm{DW}$ spin-structure transformations as well as a deviation from the linear behavior of $v(j)$, we denote this the Walker threshold current density $j_{W}$.

The $v(j)$ characteristic in Fig. 1(e) is similar to that obtained from a simulation of a DW moving in a Py wire with rough edges in Ref. 13. For simulation parameters $\beta=0.1$ and $\alpha=0.02, v$ increases linearly with $j$ up to a critical value of $j$. Beyond the critical value, vortices are periodically nucleated and annihilated in the wire and $v$ stops increasing and subsequently begins to decrease with further increase in $j$. The linear behavior differs from the ideal case $[v=(\beta / \alpha) u]$ in two ways: ${ }^{13}$ first, there is a threshold current density arising from the edge roughness and second, the gradient of $v(j)$ is reduced. These differences may be accounted for by modifying the equation for the perfect wire as follows:

$$
v=R(\beta / \alpha)\left(u-u_{c}\right) .
$$

Here, $u_{c}$ is connected to $j_{c}$ via $u_{c}=\left(P g \mu_{B} / 2 e M_{S}\right) j_{c}$ and $R$ determines the reduction in $v(j)$ compared to a perfect wire. We use Eq. (2) to fit to the measured $v(j)$ below the Walker threshold and deduce a value of $R \beta=(1.0 \pm 0.1) \times 10^{-3}$ for pure Py. Since $R<1, R \beta$ represents a lower limit for the nonadiabaticity.

Alternatively, to determine $\beta$ independently of the absolute value of the DW velocity (and thus the details of the energy landscape through which the DW travels, such as pinning sites) we can insert the measured value of $j_{W}$ into the following expression: ${ }^{12,13}$

$$
j_{W}=\frac{1}{2} \gamma H_{K} \Delta \frac{\alpha}{|\alpha-\beta|} \frac{e M_{S}}{\mu_{B} P},
$$

where $\gamma=176 \mathrm{GHz} / \mathrm{T}, H_{K}$ is the anisotropy field $\left(=2 H_{W} / \alpha=2.5 \mathrm{kOe}\right.$, where $H_{W}$ is the Walker threshold field determined by micromagnetic simulations ${ }^{24}$ ), and $\Delta$ is the effective DW width $[=20 \mathrm{~nm}$ (Ref. 2)]. For pure Py $\left[M_{S}=800 \mathrm{kA} / \mathrm{m}\right.$ and $P=0.4$ (Ref. 13) $]$ we obtain $\beta=0.13 \pm 0.01$. In contrast with previous experiments that find $\beta \sim \alpha,{ }^{2}$ we find $\beta>10 \alpha$. We also deduce that $R=(8 \pm 1) \times 10^{-3}$, thereby obtaining a measure of the extent to which obstacles to DW motion in a real wire reduce the average DW velocity. A velocity reduction of more than $100 \times$ is considerable and may not be entirely attributable to DW pinning. However, despite the number of experiments 
(a)

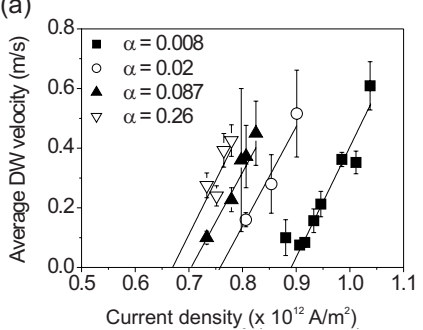

(b)

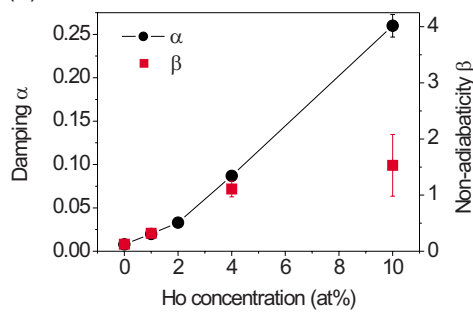

(c)

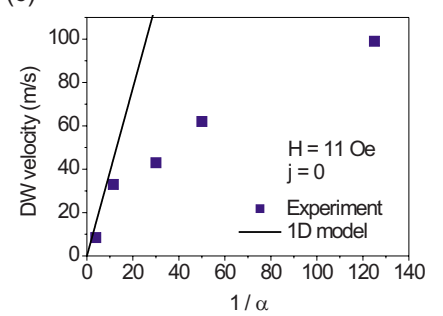

FIG. 2. (Color online) (a) Average DW velocity $v$ as a function of current density $j$ for $j \leq j_{W}$ for a 1500-nm-wide, 20-nm-thick pure Py wire and for Py wires doped with Ho. The damping $\alpha$ increases with the Ho content. The data are fitted with Eq. (2). (b) Damping $\alpha$ and nonadiabaticity $\beta$ as a function of Ho concentration. Scaling of $\beta$ and $\alpha$ occurs up to 4 at. \% Ho. (c) Average field-induced DW velocity as a function of $1 / \alpha$ determined by time-resolved MOKE (squares). The solid line is the prediction from the $1 \mathrm{D}$ model $v=(\gamma \Delta / \alpha) H$, with $\gamma=176 \mathrm{GHz} / \mathrm{T}, \Delta=20 \mathrm{~nm}$, and $H=11$ Oe.

on current-induced DW motion, the wide variation in the measured DW velocity from $<1 \mathrm{~m} / \mathrm{s}$ to $>100 \mathrm{~m} / \mathrm{s}$ is not well understood. We note that the initial state of the DW, whether it is started from rest ${ }^{6,8}$ or dynamically generated, ${ }^{4}$ seems to have an effect on whether the measured DW velocity is high or low. Meanwhile for our method of determining $\beta$ and the analysis that follows we stress that it is not the absolute DW velocities but the value of $j_{W}$ that is important.

The same experiment was performed for the Py wires doped with Ho. Figure 2(a) shows the average $v(j)$ for $j \leq j_{W}$ for wires with 1,4 , and 10 at. $\%$ Ho as well as for pure Py. Fitting the $v(j)$ with Eq. (2) we find within the experimental error the same gradient for all wire compositions. Furthermore, as $\alpha$ increases, $j_{c}$ and $j_{W}$ decrease, while the velocities remain the same, e.g., $v=0.5-0.6 \mathrm{~m} / \mathrm{s}$ at $j_{W}$ for all wires. A possible reason for the drop in $j_{c}$ is the concurrent change in $M_{S}$, a reduction in which is understood to lower the current density required for DW motion. ${ }^{25}$ The reduction in $M_{S}$ could also explain the drop in $j_{W}$ since the DW transformations that occur for $j>j_{W}$ would not require such a large spin torque effect (and hence $j$ ) in a system with reduced $M_{S}$.

Using Eq. (3) and the measured values of $j_{W}, \beta$ was determined for each wire composition. Changes in the material parameters that occur when increasing the Ho concentration were accounted for, e.g., the variation in $\alpha, M_{S}$ and $H_{K}$ (which is dominated by shape anisotropy and thus scales with $M_{S}$ ). Meanwhile the spin polarization $P$ measured by scanning-electron microscopy with polarization analysis was the same within experimental error for Ho doping up to 4 at. $\%$. The DW width $\Delta$ seen in micromagnetic simulations ${ }^{24}$ remained virtually unchanged as a function of the Ho content. $\beta$ is displayed in Fig. 2(b), alongside $\alpha$, as a function of the Ho concentration. It is seen that $\beta$ scales with $\alpha$ up to a Ho concentration of $\sim 4$ at. $\%$. The ratio $\beta / \alpha$ in this region is approximately 16 . Accounting for the uncertainty in each of the relevant parameters $\left(j_{W}, \alpha, M_{S}, H_{K}\right.$, and $P)$, the error in $\beta / \alpha$ in this region is \pm 2 . For a simple understanding of the scaling of $\beta$ and $\alpha$ we employ the definition of $\beta=\left(\lambda_{J} / \lambda_{s f}\right)^{2}$ (see Refs. 12 and 13), where $\lambda_{J}$ is the exchange length and $\lambda_{s f}$ the spin-flip length. The Ho dopant, while increasing the damping, may also be expected to act as a spin scatterer, thereby reducing $\lambda_{s f}$ and increasing $\beta$.
For Py doped with 10 at. $\%$ Ho the ratio $\beta / \alpha=6 \pm 2$. The origin of the drop in $\beta / \alpha$ between 4 and 10 at. \% Ho could be a structural transformation, which would lead to modified magnetic properties. Bailey et al. ${ }^{21}$ observed a phase transition in rare-earth-doped Py thin films from a polycrystalline to an amorphous state which is complete at 10 at. \% doping and found that it was correlated with changes in $H_{K}$. Kiessling et al. ${ }^{26}$ observed a similar behavior of $H_{K}$ as a function of the Ho concentration in Py thin films, suggesting that Py doped with 10 at. \% Ho is also amorphous. Between 4 and 10 at. $\%$ Ho, while $\alpha$ increases at the same rate as between 2 and 4 at. \% Ho, $\beta$ does not, and a possible reason for this is the reduction in spin-flip processes at grain boundaries which would accompany a transition to an amorphous state. Finally it is noted that $R$, the ratio of the measured average DW velocity and the DW velocity expected for a perfect wire, remains the same within experimental error as $\alpha$ is increased. Since $R$ is a measure of obstacles to DW motion, e.g., material defects, this may be expected as the wires were all fabricated in the same process. It should also be emphasized that, just as for the DW velocities, the value of $R$ does not affect the calculation of $\beta$ and thus the key result that $\beta$ scales with $\alpha$ for Ho doping up to 4 at. $\%$.

To show that the $\beta$ term helps to maintain $v$ for $j \leq j_{W}$ while $\alpha$ is increased, we remove spin-torque effects and induce the DW motion by field only. In this case the simplest theoretical [one-dimensional (1D)] model (Ref. 27) predicts that $v$ is inversely proportional to $\alpha[v=(\gamma \Delta / \alpha) H]$, assuming a constant DW width $\Delta$ and propagation field $H$. The average $v$ in each wire for $H=11$ Oe was measured by time-resolved MOKE and is shown in Fig. 2(c). In contrast to the currentinduced motion case discussed above, $v$ decreases as $\alpha$ is increased. However, there is agreement with the 1D model only for large values of $\alpha$. Micromagnetic simulations show that the DW motion occurs without transformations of the spin structure, allowing a comparison with the currentinduced motion below $j_{W}$. It is also seen in the simulations that, as $\alpha$ decreases, the deviation of $v$ from the 1D model prediction correlates with an increasing distortion of the VW (although the DW width does not change) so that approximation of the DW as a point-like quasiparticle becomes less and less appropriate. For example, for pure Py, the vortex core oscillates perpendicularly to the wire direction with 
$\sim 100 \mathrm{~nm}$ amplitude while the DW moves forward, emitting spin waves and this reduces the average $v$. A full discussion of the simulations will be published elsewhere.

In summary, the measurement of current-induced DW velocities in Ho-doped Py nanowires has enabled a study of the link between the Gilbert damping $\alpha$ and the nonadiabaticity of the spin transport $\beta$. To a best estimate including all sources of error we find that $\beta$ scales with $\alpha$ in the range $0-4$ at. $\%$ Ho doping. For 10 at. $\%$ doping there is a deviation from the scaling that may arise from changes in the material structure. The scaling, which is supported by the similar gradient of $v(j)$ below $j_{W}$ for all wires, as well as by theory, ${ }^{3,19}$ indicates that the angular momentum dissipation that causes damping and the spin relaxation that leads to nonadiabatic transport have a similar origin. As the damping in Ho-doped Py is thought to derive from the slow-relaxing impurity mechanism, ${ }^{22}$ a similar mechanism must also be responsible for the spin relaxation of the conduction electrons.

The ratio $\beta / \alpha \approx 16$ for low levels of Ho doping up to 4 at. \% suggests that the nonadiabatic spin torque is the dominant mechanism of current-induced DW motion in this system and predicts velocities of $60-80 \mathrm{~m} / \mathrm{s}$ just before Walker breakdown. However, in practice the DW velocities are smaller which may be due to thermal effects, material inhomogeneities, or the initial state of the DW. We determined the velocity-reduction factor $R$ to be $\sim 8 \times 10^{-3}$ for all Ho concentrations. Furthermore, since $\beta \neq \alpha$, our study could support theoretical arguments for the Gilbert rather than the Landau-Lifshitz form of damping.

In contrast to the current-induced motion where the magnitude of $v$ remains the same below the Walker threshold, the DW velocity for field-induced motion shows a strong dependence on the damping $\alpha$. In the field-driven case we measure a decreasing $v$ with increasing $\alpha$. This demonstrates how differently the underlying mechanisms of field- and currentdriven DW motion depend on the damping. It also indicates that the nonadiabatic spin torque plays a vital role in the current-induced DW propagation.

This work was supported by the Deutsche Forschungsgemeinschaft (Grants No. SPP 1133 and No. SFB513), the E.U. (Human Resources and Mobility Programme), Diamond Light Source, Didcot, U.K. [Figs. 1 and 2(a)], Elettra Synchrotron Light Source, Trieste, Italy [Fig. 2(a)], and Swiss Light Source, Paul Scherrer Institut, Villigen, Switzerland [Fig. 2(a)]. P. Möhrke thanks the Stiftung der deutschen Wirtschaft
*Present address: Institut Néel, CNRS and UJF, B.P. 166, 38042 Grenoble Cedex 9, France; t.a.moore@ physics.org

${ }^{\dagger}$ Also at Laboratory for Micro- and Nanotechnology, Paul Scherrer Institut, 5232 Villigen PSI, Switzerland.

${ }^{1}$ L. Berger, J. Appl. Phys. 55, 1954 (1984).

${ }^{2}$ G. S. D. Beach, M. Tsoi, and J. L. Erskine, J. Magn. Magn. Mater. 320, 1272 (2008).

${ }^{3}$ Y. Tserkovnyak, A. Brataas, and G. E. W. Bauer, J. Magn. Magn. Mater. 320, 1282 (2008).

${ }^{4}$ S. S. P. Parkin, M. Hayashi, and L. Thomas, Science 320, 190 (2008).

${ }^{5}$ N. Vernier, D. A. Allwood, D. Atkinson, M. D. Cooke, and R. P. Cowburn, Europhys. Lett. 65, 526 (2004).

${ }^{6}$ A. Yamaguchi, T. Ono, S. Nasu, K. Miyake, K. Mibu, and T. Shinjo, Phys. Rev. Lett. 92, 077205 (2004).

${ }^{7}$ M. Kläui, C. A. F. Vaz, J. A. C. Bland, W. Wernsdorfer, G. Faini, E. Cambril, L. J. Heyderman, F. Nolting, and U. Rüdiger, Phys. Rev. Lett. 94, 106601 (2005).

${ }^{8}$ M. Kläui, M. Laufenberg, L. Heyne, D. Backes, U. Rüdiger, C. A. F. Vaz, J. A. C. Bland, L. J. Heyderman, S. Cherifi, A. Locatelli, T. O. Mentes, and L. Aballe, Appl. Phys. Lett. 88, 232507 (2006).

${ }^{9}$ S. Yang and J. L. Erskine, Phys. Rev. B 75, 220403(R) (2007).

${ }^{10}$ L. Heyne, M. Kläui, D. Backes, T. A. Moore, S. Krzyk, U. Rüdiger, L. J. Heyderman, A. F. Rodríguez, F. Nolting, T. O. Mentes, M. Á. Niño, A. Locatelli, K. Kirsch, and R. Mattheis, Phys. Rev. Lett. 100, 066603 (2008).

${ }^{11}$ M. Kläui, J. Phys.: Condens. Matter 20, 313001 (2008).

${ }^{12}$ S. Zhang and Z. Li, Phys. Rev. Lett. 93, 127204 (2004).

${ }^{13}$ A. Thiaville, Y. Nakatani, J. Miltat, and Y. Suzuki, Europhys.
Lett. 69, 990 (2005).

${ }^{14}$ G. Tatara, H. Kohno, J. Shibata, Y. Lemaho, and K.-J. Lee, J. Phys. Soc. Jpn. 76, 054707 (2007).

${ }^{15}$ J. Xiao, A. Zangwill, and M. D. Stiles, Phys. Rev. B 73, 054428 (2006)

${ }^{16}$ M. D. Stiles, W. M. Saslow, M. J. Donahue, and A. Zangwill, Phys. Rev. B 75, 214423 (2007).

${ }^{17}$ N. Smith, Phys. Rev. B 78, 216401 (2008).

${ }^{18}$ M. D. Stiles, W. M. Saslow, M. J. Donahue, and A. Zangwill, Phys. Rev. B 78, 216402 (2008).

${ }^{19}$ I. Garate, K. Gilmore, M. D. Stiles, and A. H. MacDonald, Phys. Rev. B 79, 104416 (2009).

${ }^{20}$ O. Boulle, J. Kimling, P. Warnicke, M. Kläui, U. Rüdiger, G. Malinowski, H. J. M. Swagten, B. Koopmans, C. Ulysse, and G. Faini, Phys. Rev. Lett. 101, 216601 (2008).

${ }^{21}$ W. Bailey, P. Kabos, F. Mancoff, and S. Russek, IEEE Trans. Magn. 37, 1749 (2001)

${ }^{22}$ G. Woltersdorf, M. Kiessling, G. Meyer, J.-U. Thiele, and C. H. Back, Phys. Rev. Lett. 102, 257602 (2009).

${ }^{23}$ P. Möhrke, T. A. Moore, M. Kläui, J. Boneberg, D. Backes, S. Krzyk, L. J. Heyderman, P. Leiderer, and U. Rüdiger, J. Phys. D 41, 164009 (2008).

${ }^{24}$ T. A. Moore, P. Möhrke, L. Heyne, D. Backes, J. Rhensius, A. Kaldun, M. Kläui, U. Rüdiger, L. J. Heyderman, and J.-U. Thiele (unpublished).

${ }^{25}$ M. Yamanouchi, D. Chiba, F. Matsukura, T. Dietl, and H. Ohno, Phys. Rev. Lett. 96, 096601 (2006)

${ }^{26}$ M. Kiessling, M.S. thesis, University of Regensburg (2006).

${ }^{27}$ N. L. Schryer and L. R. Walker, J. Appl. Phys. 45, 5406 (1974). 